\title{
ENFOQUE DE LA PACIENTE EMBARAZADA CON ENFERMEDAD VALVULAR CARDIACA
}

\author{
Ana G. Múnera, Md. Cardióloga ${ }^{*}$ \\ ${ }^{1}$ Medicina Interna-Cardiología Universidad Pontificia Bolivariana, Cardiología No Invasiva Universidad CES. \\ Cardiología No Invasiva Hospital General de Medellín "Luz Castro de Gutiérrez".
}

\begin{abstract}
Resumen
Las mujeres con enfermedad valvular que están embarazadas o planean embarazarse requieren cuidadosa evaluación y manejo. El embarazo se asocia con cambios fisiológicos que pueden agravar muchas enfermedades valvulares. La mayoría de mujeres toleran bien el embarazo y el parto sin complicaciones mayores; sin embargo, algunos tipos de enfermedad valvular son mal tolerados y requieren manejo intensivo. Además las pacientes con riesgo de tromboembolismo y aquellas con prótesis valvulares requieren anticoagulación y la decisión respecto al tipo e intensidad de la anticoagulación, requiere un cuidadoso balance entre el riesgo individual de trombosis y sangrado en la madre y los peligros para el feto. La clave para optimizar los buenos resultados en las pacientes con enfermedad valvular cardiaca consiste en un diagnóstico exacto de la etiología, en la clasificación del riesgo, en la adecuada evaluación y consejería antes de la gestación y la referencia de las mujeres de alto riesgo a centros con experiencia. El manejo de estas pacientes debe ser multidisciplinario con un equipo que incluya especialistas en las áreas de obstetricia, cardiología, neonatología y anestesiología entre otros.
\end{abstract}

Palabras claves: embarazo, enfermedad valvular, prótesis valvulares, anticoagulación

\section{PREGNANT PATIENT WITH HEART VALVE DISEASE, A METHODOLOGICAL APPROACH}

\begin{abstract}
Women with valvular disease who are pregnant or plan to become pregnant require careful evaluation and management. Pregnancy is associated with physiological changes that can aggravate many valvular diseases. Most women tolerate well pregnancy and childbirth without major complications, but some types of valvular disease are poorly tolerated and require intensive management. In addition, patients who are at risk of thromboembolism and those with prosthetic valves required anticoagulation. The decision regarding the type and intensity of anticoagulation requires a careful balance between the individual risk of thrombosis, bleeding in the mother and the dangers to the fetus. The key to optimize good results in patients with valvular heart disease consists of a precise diagnosis of the etiology, classification of risk, appropriate evaluation and counseling before pregnancy and reference of high-risk women to centers with experience. The management of these patients should be multidisciplinary including specialists in the areas of obstetrics, cardiology, neonatology and anesthesiology, among others.
\end{abstract}

Keywords: pregnancy, heart valve diseases, heart valve prosthesis

Correspondencia: Ana Múnera anagm@une.net.co. Dirección Correspondencia: Calle 7B N. 27-30 Apto 1004, Medellín, Colombia. Teléfono: 310-443-07-87 384-74-54

Recibido: Febrero 3 de 2010 Aceptado: Abril 21 de 2010. 


\title{
ABORDAGEM DA PACIENTE GRÁVIDA COM DOENÇA VALVULAR CARDÍACA
}

\begin{abstract}
Resumo
As mulheres com doença valvular que estão grávidas ou pretendem engravidar necessitam de uma avaliação cuidadosa e manejo. A gravidez associase com mudanzas fisiológicas que podem piorar muitas doenças valvulares. A maioria das mulheres toleram bem a gravidez e o parto sem complicações maiores; porém alguns tipos de doença valvular são mal toleradas e precisam manejo intensivo. Alem disso as doentes com risco de tromboembolismo e aquelas com prótese valvulares precisam anticoagulação $e$ a decisão respeito ao tipo $e$ intensidade da anticoagulação, precisam um equilíbrio cuidadoso entre o risco individual de trombose e hemorragia na mãe e os perigos para o feto. A chave para otimizar os boms resultados nas doentes com doença valvular cardíaca consistem num diagnóstico exato da etiología, uma classificação do risco, na avaliação adecuada e conselhería antes da gravidez e encaminhamento de mulheres de alto risco aos centros experientes. $\mathrm{O}$ manejo destas doentes debe ser multidisciplinar com uma equipe que inclui especialistas nas áreas de obstetrícia, cardiología, neonatología e anestesiología entre outras.
\end{abstract}

Palabras-chaves: gravidez, doença valvular, prótese valvular, anticoagulação

\section{Introducción}

Aunque la prevalencia de la enfermedad materna cardíaca clínicamente significativa durante el embarazo es baja (menos del 1\%) (1), su presencia incrementa el riesgo de eventos adversos maternos, fetales y neonatales (2). La enfermedad valvular es la principal causa de enfermedad cardíaca durante el embarazo en los países en desarrollo y la segunda causa en países occidentales luego de las enfermedades cardíacas congénitas.

Hay dos grupos de mujeres embarazadas con la enfermedad valvular: uno está formado por mujeres que saben que tienen la enfermedad y que consultan antes del embarazo para conocer los potenciales riesgos para la madre y el feto y el otro, por mujeres que son diagnosticadas durante la gestación. En los dos grupos los efectos de la lesión valvular pueden ser exacerbados por los cambios fisiológicos normales del embarazo. Sin embargo, con una adecuada evaluación y manejo, la mayoría de mujeres pueden terminar su gestación satisfactoriamente. La clave para optimizar los buenos resultados en las pacientes con enfermedad valvular cardíaca consiste en un diagnóstico exacto de la etiología, en una clasificación del riesgo, en una adecuada evaluación y consejería antes de la gestación y en la referencia de las mujeres de alto riesgo a centros con experiencia. La anticoagulación está indicada en algunos casos tales como el riesgo tromboembólico y prótesis valvulares mecánicas y la decisión con relación al tipo e intensidad de anticoagulación depende de la indicación y los riesgos potenciales para la madre y el feto $(4,5)$.

El manejo de estas pacientes debe ser multidisciplinario con un equipo que incluya especialistas en las áreas de obstetricia, cardiología, neonatología y anestesiología entre otros. En este artículo se revisa los cambios hemodinámicos del embarazo, clasificación, evaluación y manejo de mujeres con enfermedad valvular que planean embarazarse o están embarazadas.

\section{Cambios hemodinámicos del embarazo}

La evaluación y el manejo de la mujer embarazada con enfermedad valvular requieren la comprensión de los cambios fisiológicos en la gestación, en el trabajo de parto, en el parto y en el período inmediato post parto. El volumen sanguíneo se incrementa a partir de la sexta semana de gestación, aumenta rápidamente hasta la mitad del embarazo y luego el incremento continúa pero con menor velocidad. La expansión de volumen en promedio es del $50 \%$. Debido a que el incremento en el volumen plasmático es más rápido que el incremento de la masa de eritrocitos, la concentración de hemoglobina disminuye, presentándose la anemia fisiológica del embarazo. El incremento en el volumen sanguíneo se acompaña de aumento en 
el gasto cardíaco, y por lo tanto del volumen latido. Durante el tercer trimestre de gestación la posición del cuerpo influencia el gasto cardíaco, que aumenta en la posición decúbito lateral y disminuye en la posición supina debido a la compresión por el útero de la cava inferior, con reducción del retorno venoso al corazón (6-9).

La frecuencia cardíaca durante el tercer trimestre se incrementa entre 10 y 15 latidos por minuto. La presión arterial sistémica comienza a descender durante el primer trimestre, alcanza la máxima disminución en la mitad del embarazo y vuelve al nivel normal antes del parto. La disminución en la presión arterial es más marcada en la presión diastólica y es debido a la disminución de la resistencia vascular sistémica por reducción en el tono vascular. Durante el trabajo de parto y en el parto, el consumo de oxígeno aumenta tres veces y la presión arterial sistólica y diastólica se incrementa durante las contracciones. En el post parto ocurre un aumento en el retorno venoso debido a la mejoría de la compresión de la vena cava inferior y al traslado de sangre del útero a la circulación sistémica. Este cambio en el volumen sanguíneo ocurre a pesar de las pérdidas sanguíneas del parto y puede ocasionar incremento en la presión de llenado del ventrículo izquierdo, en el volumen latido y en el gasto cardíaco. Los cambios hemodinámicos del embarazo persisten durante el post parto y gradualmente retornan a los niveles basales en 12- 24 semanas luego del parto (6-9).

La gestación se asocia a un estado hipercoagulable debido a la reducción relativa de la actividad de la proteína $\mathrm{C}$ y $\mathrm{S}$, estasis e hipertensión venosa. Los estrógenos interfieren con el depósito de colágeno en la capa media de las arterias musculares y la elastasa circulante puede romper la lámina elástica y debilitar la capa media de la aorta durante la gestación. El debilitamiento de la pared vascular puede predisponer a disección en presencia o no de enfermedad del tejido conectivo. La relaxina y la hormona factor del crecimiento similar a la insulina, se detectan en sangre durante la gestación y causan reducción en la síntesis de colágeno y predisponen a la disección aórtica durante el embarazo (10-12).

\section{Prevalencia de enfermedad valvular en mujeres en edad reproductiva}

La enfermedad cardíaca reumática continúa siendo prevalente en los países en desarrollo donde es responsable de hasta el $90 \%$ de las enfermedades cardíacas durante la gestación (5). El desarrollo de tratamientos efectivos en la infancia y la niñez contra la fiebre reumática ha resultado en un incremento en la prevalencia de enfermedad valvular congénita en mujeres en edad reproductiva. Otras causas de enfermedad valvular son el prolapso valvular mitral (PVM), endocarditis previa y enfermedad valvular asociada a enfermedades sistémicas. Las prótesis valvulares pueden encontrarse en pacientes jóvenes y debe tenerse en cuenta la necesidad de anticoagulación en las prótesis mecánicas y la posibilidad de deterioro de las prótesis las biológicas durante la gestación. Para la evaluación de la enfermedad valvular en mujeres en edad reproductiva, se deben clasificar de acuerdo al impacto hemodinámico (estenosis vs. regurgitación) y válvula comprometida, Tabla 1 (3).

TABLA 1. Clasificación de la enfermedad valuular en mujeres en edad reproductiva (3)

\begin{tabular}{|c|}
\hline ESTENOSIS VALVULAR \\
\hline $\begin{array}{l}\text { ESTENOSIS AÓRTICA } \\
\text { - Congénita. } \\
\text { - Reumática. } \\
\text { - Recurrente luego de valvulotomía en la infancia. }\end{array}$ \\
\hline ESTENOSIS MITRAL \\
\hline $\begin{array}{l}\text { ESTENOSIS PULMONAR } \\
\text { - Congénita asociada a otras alteraciones. } \\
\text { - Estenosis valvular pulmonar aislada. }\end{array}$ \\
\hline INSUFICIENCIA VALVULAR \\
\hline $\begin{array}{l}\text { INSUFICIENCIA AÓRTICA } \\
\text { - Congénita } \\
\text { - Reumática } \\
\text { - Asociada a Enfermedad del Tejido conectivo ( Síndrome de } \\
\text { Marfan) }\end{array}$ \\
\hline $\begin{array}{l}\text { INSUFICIENCIA MITRAL } \\
\text { - Prolapso valvular mitral. } \\
\text { - Reumática } \\
\text { - Congénita }\end{array}$ \\
\hline $\begin{array}{l}\text { INSUFICIENCIA PULMONAR } \\
\text { - Residual luego de intervención de tetralogía de Fallop o } \\
\text { estenosis pulmonar }\end{array}$ \\
\hline
\end{tabular}

\section{Evaluación diagnóstica}

La evaluación inicial de la mujer con enfermedad valvular que está planeando embarazarse o que ya está 
embarazada se basa en la historia clínica y el examen físico. La historia clínica debe incluir todos los estudios diagnósticos realizados previamente incluyendo ecocardiografías, pruebas de estrés y datos de cualquier intervención realizada previamente. La tolerancia al ejercicio inicial es un predictor importante de la capacidad para tolerar la gestación, independiente del tipo de lesión valvular. Muchos de los síntomas normales del embarazo, son comunes a los síntomas de descompensación cardíaca. La disnea con el ejercicio, la ortopnea, el edema de tobillos y las palpitaciones pueden presentarse durante la gestación, pero la presencia de angina, disnea de reposo, disnea paroxística nocturna o arritmia sostenida son hallazgos patológicos y ameritan una evaluación completa. En el examen físico de las maternas normales puede encontrarse taquicardia leve en reposo, pulsos saltones, aumento de la presión del pulso con presión sistólica normal baja y extremidades calientes. El impulso precordial es hipercinético y el primer ruido puede ser un poco más intenso de lo normal, con desdoblamiento. El segundo ruido también puede encontrarse fisiológicamente desdoblado, pero puede ser amplio y aparecer fijo al final de la gestación. Un tercer ruido está presente en la mayoría de pacientes $(3,13,14)$.

Los soplos durante la gestación típicamente son suaves (grado I ó II), localizados en la región pulmonar y nunca acompañados de soplos diastólicos o signos de falla cardíaca. Puede auscultarse soplos continuos normales como el soplo venoso cervical (más audible en la fosa supraclavicular derecha y puede desaparecer con el movimiento del mentón hacia el estetoscopio o la presión digital en la vena yugular ipsilateral ) y el soplo mamario que puede ser continuo ó sistólico, y puede desaparecer con la presión del diafragma del estetoscopio sobre la mama (3,13-15).

\section{Electrocardiograma (ECG)}

Durante el embarazo, en el ECG puede presentarse desviación del eje del QRS, onda $\mathrm{Q}$ pequeña y onda $T$ invertida en DIII, taquicardia sinusal y cambios en el segmento ST y la onda T, así como latidos auriculares y ventriculares prematuros $(13,16)$.

\section{Radiografía de Tórax}

No se realiza rutinariamente en las embarazadas debido a la radiación, pero debe considerarse en pacientes con sospecha de compromiso cardíaco o inicio reciente de disnea o falla cardíaca. En ella puede observarse rectificación del borde cardíaco superior izquierdo, posición horizontal del corazón, incremento de la trama pulmonar y derrame pleural pequeño en el período post parto $(13,16)$.

\section{Ecocardiografía}

En el embarazo normal puede evidenciarse incremento leve en las dimensiones ventriculares y son frecuentes la insuficiencia pulmonar y la tricuspídea leve. Puede presentarse insuficiencia mitral "fisiológica" en ausencia de alteración estructural de la válvula y las insuficiencias auriculo-ventriculares pueden ser debidas a la dilatación del anillo que acompaña el aumento del tamaño ventricular. Puede encontrarse un derrame pericárdico pequeño en el $25 \%$ de las pacientes. El incremento del gasto cardíaco durante el embarazo lleva a un incremento en las velocidades doppler de los flujos transvalvulares, por lo tanto es indispensable la medición de los orificios valvulares en casos de estenosis y la cuantificación de las insuficiencias para una evaluación más exacta del compromiso y severidad de la enfermedad valvular. El ultrasonido no posee riesgo de radiación en la madre ni en el feto $(13,16,17)$.

\section{Consideraciones generales en el manejo de mujeres con enfermedad valvular}

El paso inicial en la evaluación de la mujer con enfermedad valvular es establecer el nivel de riesgo materno y fetal durante la gestación. En la Tabla 2 se presentan las lesiones valvulares cardíacas asociadas con riesgo materno y/o fetal alto durante la gestación $(3,14,18)$.

TABLA 2. Lesiones valvulares cardíacas asociadas con riesgo Materno y/o fetal alto durante la gestación.

1. Estenosis aórtica severa con o sin síntomas

2. Insuficiencia Aórtica con síntomas Clase NY III-IV.

3. Estenosis Mitral con síntomas Clase NY II-IV

4. Insuficiencia Mitral con síntomas Clase NY III-IV

5. Enfermedad valvular mitral o aórtica con hipertensión pulmonar severa (Presión pulmonar > del 75\% de la presión sistémica).

6. Enfermedad valvular aórtica o mitral con disfunción ventricular izquierda severa (Fracción de expulsión $<40 \%$ )

7. Prótesis valvular mecánica que requiere anticoagulación.

8. Síndrome de Marfan con o sin insuficiencia aórtica.

9. Cianosis Materna

10. Clase Funcional de NY III ó IV.

En pacientes con riesgo alto que se presentan para evaluación antes de embarazarse, se recomienda tratamiento 
especialmente si la lesión valvular permite intervención percutánea o reparo quirúrgico. Cuando el cambio valvular es la única opción, muchos médicos y pacientes prefieren prótesis biológicas, a pesar de su limitada durabilidad, para evitar los riesgos de la anticoagulación durante la gestación. Sin embargo, debe analizarse con la paciente y su familia claramente las ventajas y desventajas de utilizar prótesis biológicas vs mecánicas (3).

\section{Evaluación antes del Embarazo}

El manejo de las pacientes con enfermedad valvular debe realizarse idealmente antes de la concepción. Es necesario un examen físico cuidadoso y evaluación de la capacidad funcional para determinar la posibilidad de tolerar los cambios hemodinámicos del embarazo y el riesgo de complicaciones durante la gestación. Debe incluir un electrocardiograma de doce derivaciones y una ecocardiografía doppler. En pacientes con sospecha de compromiso de la capacidad funcional debe realizarse una prueba de ejercicio, idealmente con medición del consumo de oxígeno. Deben suspenderse las medicaciones con riesgo potencial para el feto $(2,13,19,20)$.

\section{Manejo durante la gestación}

El tipo y frecuencia de los controles durante la gestación está determinado por las características de la lesión valvular y comportamiento clínico de cada paciente. En general los controles deben realizarse cada mes en pacientes con enfermedad leve y cada dos semanas en pacientes con enfermedad moderada y severa hasta la semana 28-30 de gestación y luego semanalmente hasta el parto. Es importante educar las pacientes sobre los signos y síntomas de descompensación cardíaca. Aunque la gestación puede inicialmente ser bien tolerada, puede ocurrir incremento en las demandas cardíacas por anemia, arritmias, embolismo, dolor y ansiedad. En estos casos además de corregir el factor desencadenante, las demandas cardíacas pueden disminuirse con reposo en cama y oxígeno. En ocasiones se requiere tratamiento médico para controlar la frecuencia cardíaca y mejorar las condiciones de pre y post carga. Las lesiones dependientes de precarga se pueden beneficiar de posición en decúbito lateral, para prevenir la reducción del retorno venoso por la compresión de la vena cava inferior por el útero grávido (13).

Cuando es necesario el uso de medicaciones se debe emplear la menor dosis terapéutica conocida que sea segura para el feto. Algunas medicaciones están contraindicadas durante el embarazo como inhibidores de la enzima convertidora de la angiotensina (IECAS), bloqueadores del receptor de la angiotensina, amiodarona y nitroprusiato. Medicaciones que pueden ser utilizadas durante la gestación incluyen betabloqueadores, hidralazina, diuréticos y digoxina (21).

\section{Trabajo de parto y parto}

El momento y modo del parto debe ser discutido en forma conjunta entre el obstetra, cardiólogo y anestesiólogo obstétrico. En general, el parto vaginal con adecuada anestesia y acortamiento de la segunda etapa es seguro y puede realizarse en la mayoría de pacientes con enfermedad valvular. La cesárea está asociada con mayor número de complicaciones y es usualmente realizada por indicaciones obstétricas y en ocasiones en el paciente con inestabilidad hemodinámica (22-24).

Las indicaciones de cesárea son las mismas para las mujeres con enfermedad valvular y la población general. Se benefician de cesárea las pacientes con las siguientes características: endocarditis que requiere un reemplazo valvular urgente al final del embarazo, mujeres con restricción severa del crecimiento intrauterino, Eisenmenger, condiciones no favorables del cuello uterino, estenosis aórtica severa, dilatación aórtica o disección, historia reciente de Infarto agudo del miocardio y descompensación aguda severa durante el trabajo de parto (5).

El monitoreo hemodinámico durante el trabajo de parto y el parto se recomienda en pacientes sintomáticas $y$ en pacientes con estenosis valvulares moderadas $y$ severas, disfunción ventricular izquierda e hipertensión pulmonar. Las pacientes con estenosis valvular severa del lado izquierdo frecuentemente requieren monitoreo hemodinámico con evaluación de la saturación de oxígeno, electrocardiograma, presión arterial, evaluación de la presión pulmonar, presión en cuña y gasto cardíaco. También es necesario el monitoreo fetal debido a que el estrés fetal es un indicador del compromiso del gasto cardíaco (13).

\section{Puerperio inmediato}

A pesar de las pérdidas sanguíneas presentadas durante el parto, el puerperio temprano presenta incremento del retorno venoso al corazón por el paso de sangre 
del útero a la circulación sistémica, reducción de la compresión de la vena cava y movilización de líquido desde la parte inferior del cuerpo. Estos cambios pueden llevar a descompensación y se requiere monitoreo hemodinámico por doce a 24 horas (25).

\section{Profilaxis antibiótica}

El comité de Fiebre Reumática, Endocarditis y Enfermedad de Kawasaki de la Asociación Americana del Corazón no recomiendan el uso rutinario de profilaxis antibiótica en pacientes con enfermedad valvular que tienen parto vaginal o cesárea no complicadas y no se sospecha infección. Los antibióticos son opcionales en pacientes con alto riesgo como pacientes con prótesis valvulares, endocarditis previa, enfermedad cardíaca congénita compleja y conductos sistémico-pulmonares creados quirúrgicamente (26-28). Sin embargo, algunos reportes han informado bacteremia luego del trabajo de parto y parto entre el $10-20 \%$ de las pacientes. Estos reportes han llevado a algunos autores a recomendar dar antibióticos ante la dificultad de predecir partos complicados, el bajo costo de la terapia y las complicaciones devastadoras de la endocarditis. El esquema recomendado es ampicilina 2 g IV o IM más gentamicina $1,5 \mathrm{mg} / \mathrm{kg}$ (sin exceder $120 \mathrm{mg}$ ) al inicio del trabajo de parto o dentro de los treinta minutos de iniciada la cesárea y seis horas luego ampicilina $1 \mathrm{~g}$ IV o IM ó amoxacilina $1 \mathrm{~g}$ VO. En pacientes alérgicos a la penicilina se recomienda el uso de vancomicina más gentamicina (29-31).

\section{Lesiones valvulares específicas}

\section{Estenosis mitral}

Es la lesión valvular más común durante el embarazo y es debida en la mayoría de casos a enfermedad reumática. El gradiente a través de la válvula puede aumentar durante la gestación por el aumento de la frecuencia cardíaca y el volumen latido, lo cual aumenta la presión auricular izquierda y desarrolla o empeora los síntomas. El incremento en la presión auricular puede también ocasionar arritmias auriculares. Aún en mujeres previamente asintomáticas, el acortamiento del período de llenado diastólico debido a fibrilación auricular o enfermedades asociadas puede ocasionar descompensación hemodinámica.

El pronóstico materno es favorable en maternas con estenosis leve cuando se compara con los controles.
La morbilidad materna es mayor en pacientes con estenosis moderada y severa, pueden desarrollar falla cardíaca, arritmias (especialmente fibrilación auricular) y requerir inicio de medicación y hospitalización. La incidencia de complicaciones maternas está relacionada con la severidad de la estenosis y se presentan en el $67 \%$ de mujeres con estenosis severa, $38 \%$ de las mujeres con estenosis moderada y $26 \%$ en estenosis leve. La presencia de complicaciones maternas se asocia con la clase funcional de NY y severidad de la estenosis antes de la gestación. A pesar de la alta morbilidad reportada en pacientes con estenosis mitral, la mortalidad es rara. En cuanto al pronóstico fetal la severidad de la estenosis mitral se relaciona con partos pretérmino, retardo en el crecimiento intrauterino y bajo peso al nacer. Se recomienda el seguimiento estrecho de las pacientes con estenosis mitral severa, aún si están asintomáticas antes del embarazo y primer trimestre de la gestación. El control ecocardiográfico debe realizarse al tercer y quinto mes, y luego cada mes para evaluar los gradientes transvalvulares y presión pulmonar $(3,13,14,17,29,32)$.

\section{Tratamiento de la estenosis mitral}

Las mujeres jóvenes con historia de fiebre reumática aguda y carditis deben continuar recibiendo profilaxis para fiebre reumática con penicilina, así como las mujeres no embarazadas.

Cuando se presentan síntomas debido a descompensación de la estenosis mitral, los objetivos del tratamiento son: 1- Tratar y corregir cualquier condición desencadenante como infección, fiebre ó anemia, 2- Disminuir la frecuencia cardíaca para prolongar el tiempo de llenado diastólico, 3- Disminuir la precarga si se presenta edema pulmonar y 4- Conservar la presión arterial.

Las pacientes con estenosis leve a moderada pueden ser manejadas con restricción de la actividad física, restricción de líquidos y de sal, y administración de betabloqueadores. Los betabloqueadores son la principal estrategia de manejo, son utilizados para prevenir y tratar la taquicardia, optimizar el tiempo de llenado diastólico y son relativamente seguros y bien tolerados por la madre y el feto. Se usan betabloqueadores cardioselectivos como el metoprolol y atenolol; se prefiere el metoprolol, debido a que el atenolol se ha asociado con retardo en el crecimiento intrauterino. Los diuréticos deben evitarse en el primer trimestre del 
embarazo y tener precaución por el riesgo de producir déficit de volumen e hipoperfusión uteroplacentaria. Los diuréticos de asa son útiles en el post parto inmediato en pacientes que desarrollan edema pulmonar secundario al incremento súbito del volumen intravascular o al inicio de fibrilación auricular. En pacientes con fibrilación auricular puede utilizarse digoxina o betabloqueadores para reducir la frecuencia cardíaca.

La mayoría de pacientes con estenosis moderada a severa (área valvular mitral $<1,5 \mathrm{~cm}^{2}$ ) presentan empeoramiento en una o dos clases funcionales durante el tercer trimestre de gestación y/o trabajo de parto y el parto (figura 1). Cuando el compromiso hemodinámico persiste a pesar de tratamiento médico adecuado,

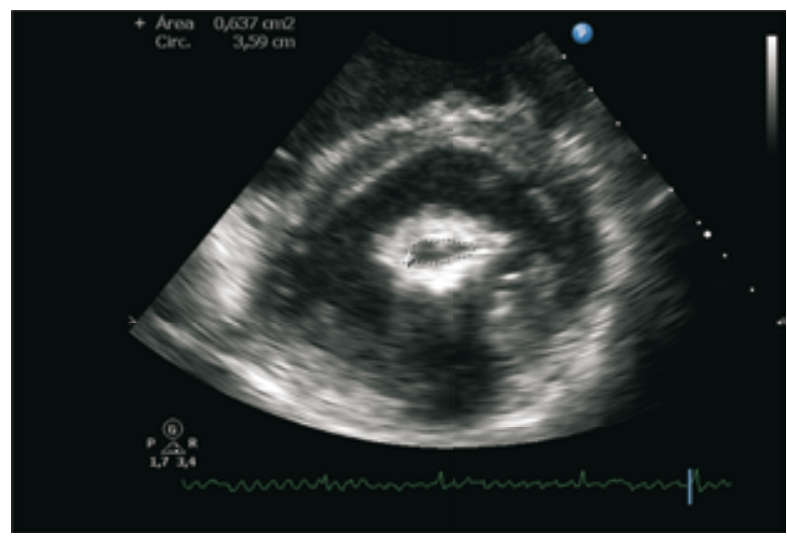

FIGURA 1. Ecocardiografía de una paciente con estenosis mitral severa con área valuular por planimetría de 0,63 $\mathrm{cm}^{2}$. (Imagen Servicio Cardiología No Invasiva, Hospital General de Medellín).

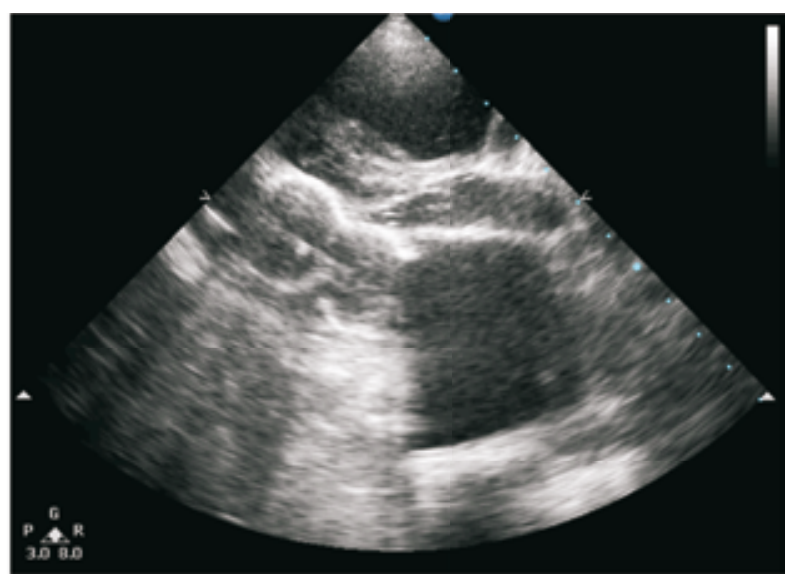

FIGURA 2. Imagen ecocardiográfica durante la dilatación percutánea de la válvula mitral con balón de Inoue. (Imagen Servicio Cardiología No Invasiva, Hospital General de Medellín). y los pacientes con estenosis mitral severa desarrollan clase funcional de NY III-IV, puede ser necesaria la valvulotomía percutánea con balón (figura 2). Este procedimiento debe evitarse durante el primer trimestre de gestación, y recurrir a él luego que las medidas de tratamiento médico óptimo han fallado. Cuando se realice este procedimiento debe ser en centros con experiencia y bajo la guía de ecocardiografía (figuras 3 y 4). Es indispensable una adecuada protección pélvica, abdominal y disminuir al máximo el tiempo de fluoroscopia para evitar la radiación del feto $(3,4$, $14,17,29,33,34)$.

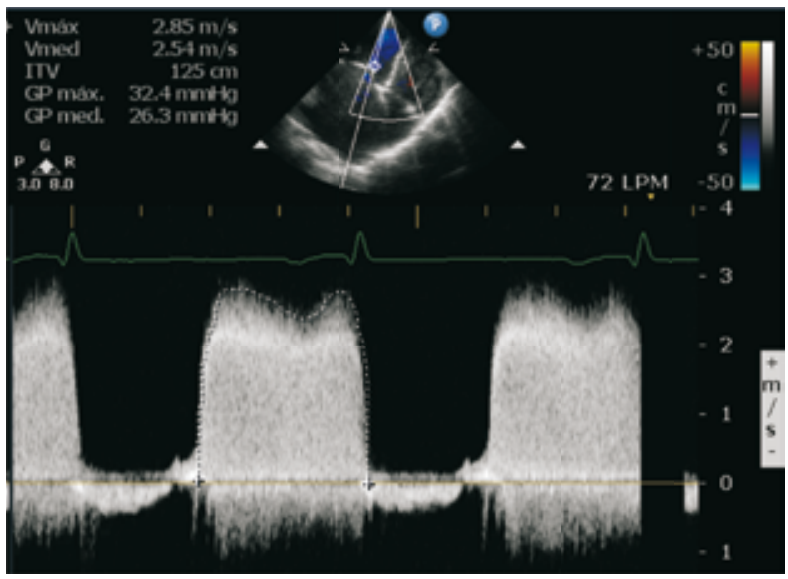

FIGURA 3. Doppler continuo de la válvula mitral, se aprecia el incremento severo en los gradientes transvalvulares mitrales (gradiente máximo de $32 \mathrm{mmHg}$ y medio de $26 \mathrm{mmHg}$ ). (Imagen Servicio Cardiología No Invasiva, Hospital General de Medellín).

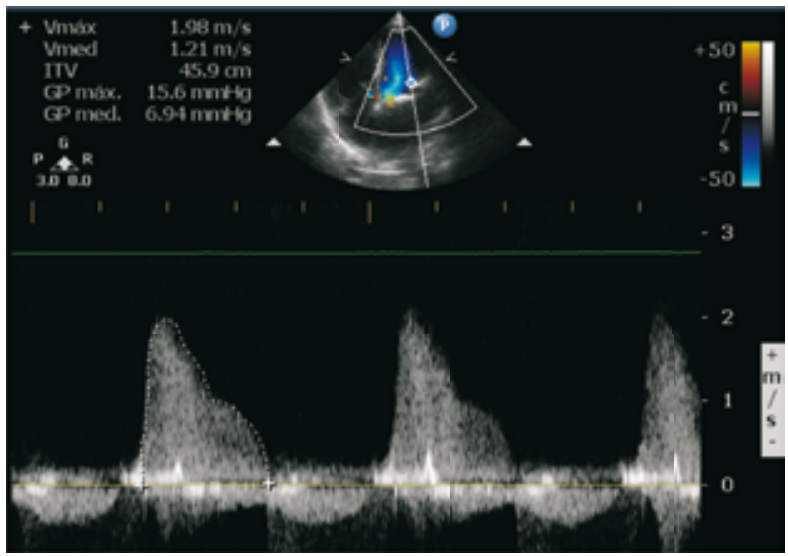

FIGURA 4. Se observa con el doppler continuo de la válvula mitral la reducción de los gradientes transvalvulares en relación con los iniciales (gradiente máximo de $15,6 \mathrm{mmHg}$ y medio de $6,9 \mathrm{mmHg}$ ). (Imagen Servicio Cardiología No Invasiva, Hospital General de Medellín). 
Cuando la paciente tiene insuficiencia mitral significativa asociada a la estenosis mitral o existe contraindicación para la valvulotomía mitral percutánea, puede ser necesaria la cirugía de la válvula mitral en casos extremos de descompensación a pesar de tratamiento médico agresivo La cirugía cardiaca debe evitarse durante la gestación siempre que sea posible, aunque existe reporte de procedimientos exitosos. El riesgo de muerte fetal durante cirugía cardíaca abierta se ha estimado entre $20-30 \%$ y es impredecible. La valvulotomía mitral cerrada ha sido reportada especialmente en países en desarrollo como un procedimiento seguro para la madre y con una mortalidad fetal entre $2-12 \%$. Actualmente la valvulotomía mitral percutánea ha reemplazado la cirugía y es considerada el procedimiento valvular de elección cuando se requiera y la anatomía sea favorable $(14,17,29,35,36)$.

El parto vaginal puede ser permitido en la mayoría de pacientes con estenosis mitral. La segunda etapa del trabajo del parto debe ser acortada con el uso de forceps o vacuum extractor. La anestesia epidural es la forma de analgesia más apropiada en pacientes con estenosis mitral; esta forma de anestesia se asocia a disminución en la presión pulmonar y auricular debido a vasodilatación sistémica. En pacientes con estenosis moderada a severa se recomienda monitoreo hemodinámico durante el trabajo de parto y parto para optimizar el tratamiento y evitar el aumento en la presión auricular izquierda $(14,17,29)$.

\section{Insuficiencia mitral}

El prolapso valvular mitral es la principal causa de insuficiencia mitral en mujeres embarazadas y ha sido reportada en el 2,4\% de la población general y en 1,2\% de las mujeres embarazadas (figura 5). La mayoría de pacientes son asintomáticas; el diagnóstico se presenta cuando se ausculta un soplo sistólico tardío precedido por un click y se confirma por ecocardiografía. Esta patología es bien tolerada durante la gestación, ya que el aumento del volumen cardíaco disminuye el prolapso y la disminución de la resistencia sistémica reduce la severidad de la insuficiencia mitral. Otra etiología de insuficiencia mitral en nuestro medio es la fiebre reumática, usualmente asociada a algún grado de estenosis.

Esta patología es usualmente bien tolerada durante el embarazo, incluso si es severa. En las pacientes asintomáticas no está indicado ningún tipo de tratamiento

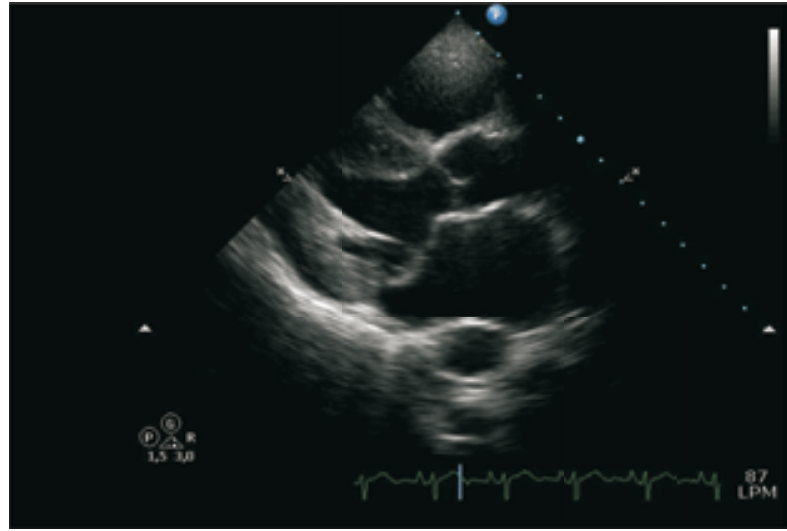

FIGURA 5. Paciente con prolapso de la valva anterior de la válvula mitral. (Imagen Servicio Cardiología No Invasiva, Hospital General de Medellín).

farmacológico y las que presentan arritmias sintomáticas pueden recibir betabloqueadores; en pacientes con síntomas debidos a congestión pulmonar está indicado el uso de diuréticos y cuando existe compromiso de la función ventricular izquierda, se puede emplear digital. El uso de vasodilatadores está indicado sólo en casos de hipertensión arterial concomitante. La hidralazina ha demostrado ser segura durante el embarazo y puede emplearse para disminuir la carga ventricular y prevenir el deterioro hemodinámico asociado al trabajo de parto. Otros vasodilatadores de utilidad son los nitratos; no deben utilizarse IECAS ni antagonistas del receptor de la angiotensina debido a sus efectos en el feto.

La insuficiencia mitral asociada a PMV (figura 6) puede ser tratada médicamente durante la gestación; raras veces amerita manejo quirúrgico debido a la ruptura aguda de las cuerdas tendinosas y al empeoramiento severo de una lesión regurgitante. Debido al riesgo de pérdida fetal, la cirugía debe evitarse durante el embarazo y considerarse solo en pacientes con falla cardíaca severa y compromiso hemodinámico a pesar del tratamiento médico adecuado. Cuando esté indicada la cirugía se prefiere el reparo valvular $(14,17,29,37,38)$.

\section{Estenosis aórtica}

La causa más común de estenosis aórtica en la mujer embarazada es la enfermedad valvular aórtica congénita. La estenosis aórtica de etiología reumática es menos común y se asocia a compromiso mitral. Las pacientes con obstrucción leve y función ventricular izquierda normal pueden manejarse en forma conservadora; las pacientes con estenosis moderada a 


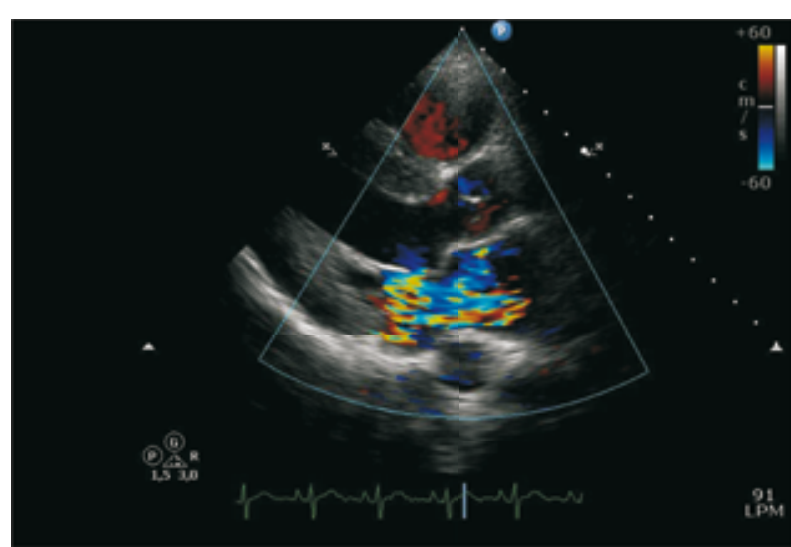

FIGURA 6. Insuficiencia mitral severa originada por la presencia de prolapso de la valva anterior de la mitral. (Imagen Servicio Cardiología No Invasiva, Hospital General de Medellín).

severa o síntomas deben aplazar la concepción hasta que se haya intervenido. La estenosis aórtica severa se ha asociado a morbilidad materna y pronóstico fetal desfavorable, aunque la mortalidad materna es baja.

Las mujeres embarazadas con estenosis aórtica severa que están asintomáticas o con síntomas leves pueden ser manejadas en forma conservadora con reposo en cama, oxígeno y betabloqueadores; en presencia de signos de congestión pulmonar y falla pueden emplearse diuréticos con precaución. En pacientes con estenosis aórtica severa y síntomas severos de descompensación, sino existe respuesta al tratamiento médico, debe considerarse la posibilidad de tratamiento percutáneo con valvulotomía aórtica percutánea o cirugía (según las características anatómicas) antes del trabajo de parto y parto. Aunque este tipo de intervenciones son difíciles y con riesgo para el feto y la madre, se han reportado casos exitosos.

En el caso de pacientes que presentan síntomas severos durante el embarazo, no hay respuesta al tratamiento médico y no pueden tener el parto (feto sin madurez), pueden requerir valvuloplastia con balón ó cirugía. En ocasiones se prefiere la valvuloplastia con balón por tener menos riesgo de pérdida fetal comparada con la intervención quirúrgica. Cuando algún un tipo de intervención está indicada y la madurez fetal está confirmada, se puede tener el parto primero y realizar el reemplazo o reparo quirúrgico inmediatamente. Existe asociación entre aorta bicúspide y dilatación de la raíz aórtica, que puede predisponer a disección aórtica espontánea en el tercer trimestre, especialmente si tiene asociada coartación aórtica.
La forma preferida del parto es el vaginal con acortamiento de la segunda fase del trabajo de parto. La anestesia regional para el trabajo de parto y el parto debe ser utilizada con precaución en pacientes con estenosis aórtica, para evitar la reducción en la resistencia vascular sistémica. La anestesia general es la técnica preferida cuando existe indicación de realizar cesárea en pacientes con estenosis aórtica $(3,4,14,17,29,39,40-42)$.

\section{Insuficiencia aórtica}

La insuficiencia aórtica en mujeres jóvenes puede ser debida a aorta bicúspide, enfermedad reumática, endocarditis o dilatación del anillo. La insuficiencia aórtica sin disfunción ventricular es bien tolerada durante la gestación probablemente debido a la disminución del volumen regurgitante, por la reducción de la resistencia vascular sistémica y el aumento de la frecuencia cardíaca que acorta el tiempo diastólico. Durante el trabajo de parto y el parto pueden empeorar la insuficiencia aórtica debido al incremento del retorno venoso y la resistencia vascular $(3,4,14,17)$.

En casos de insuficiencia aórtica severa con disfunción ventricular izquierda y síntomas, debe iniciarse terapia médica que incluye restricción de sal, diuréticos y digoxina. Los vasodilatadores como la hidralazina y nitratos pueden utilizarse en lugar de los IECAS, lo cuales están contraindicados. Estas pacientes sintomáticas y con disfunción ventricular izquierda se benefician de monitoreo hemodinámico durante el trabajo de parto y parto (figura 7) (43). Si la cirugía está indicada, debería si es posible realizarse luego del parto por el riesgo de pérdida fetal. La cirugía durante la gestación debe considerarse sólo para el control de síntomas refractarios clase funcional de NY III o IV. Las recomendaciones de cambio valvular aórtico basados en el tamaño ventricular no son utilizados en la pacientes embarazadas $(3,4,14,17,29)$.

\section{Estenosis pulmonar}

La estenosis pulmonar aislada durante la gestación es debida a obstrucción congénita de la válvula, pero también puede ocurrir a nivel sub o supravalvular ó como consecuencia del deterioro del homoinjerto implantado durante un procedimiento de Ross previo; también puede estar asociada a otros defectos cardíacos congénitos como la tetralogía de Fallop. La estenosis pulmonar aislada, incluso cuando es 


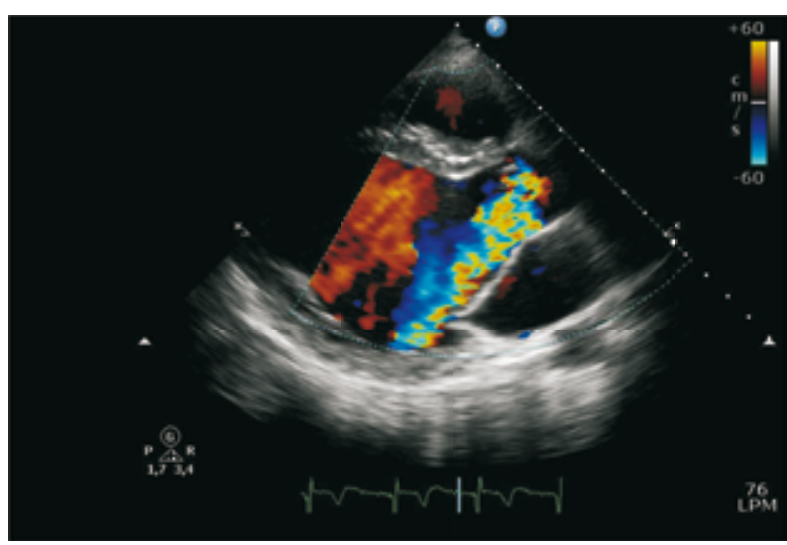

FIGURA 7. Paciente con insuficiencia aórtica y dilatación ventricular. (Imagen Servicio Cardiología No Invasiva, Hospital General de Medellín).

severa es bien tolerada durante la gestación. Aunque el reporte en la literatura es limitado, la información disponible indica que el embarazo en pacientes con estenosis pulmonar es tolerado bien, y en contraste con la estenosis mitral y estenosis aórtica, la severidad de la estenosis pulmonar no afecta el pronóstico materno o fetal $(3,4,14,17,29)$.

La valvuloplastia con balón se recomienda en pacientes no embarazadas cuando el gradiente en el tracto de salida del ventrículo derecho es mayor de $50 \mathrm{~mm}$ $\mathrm{Hg}$ en reposo o cuando la paciente es sintomática; este procedimiento rara vez está indicado durante el embarazo en pacientes que están asintomáticas o levemente sintomáticas antes del embarazo. El parto vaginal es bien tolerado y puede permitirse en la gran mayoría de pacientes $(3,4,14,17,29)$.

\section{Insuficiencia pulmonar}

Puede ser ocasionada por la enfermedad cardiaca congénita y más a menudo como secuela de un procedimiento previo como corrección de Tetralogía de Fallop $(44,45)$; estas pacientes tienen mayor riesgo de arritmias. La insuficiencia pulmonar es bien tolerada si la paciente es asintomática y tiene función y dimensiones normales del ventrículo derecho; las sintomáticas y con ventrículos derechos aumentados de tamaño o con disfunción podrían beneficiarse del reemplazo valvular antes de la gestación. La presencia de síntomas durante el embarazo puede manejarse con diuréticos y antiarrítmicos cuando sea necesario $(3,4,14,17,29)$.

\section{Enfermedades de la válvula tricúspide}

Las enfermedades de la válvula tricúspide pueden ser congénitas (anomalía de Ebstein y atresia tricuspídea) o adquiridas (endocarditis, enfermedad mixomatosa y carcinoide). La insuficiencia tricuspídea aislada no ocasiona problemas significativos durante la gestación, y debe tenerse precaución al emplear diuréticos por el riesgo de hipoperfusión placentaria. La estenosis tricuspídea es rara durante la gestación, aunque se han reportados casos exitosos de valvuloplastia percutánea con balón (14).

\section{Síndrome de Marfan}

El síndrome de Marfan es un trastorno hereditario del tejido conectivo debido a anormalidades del gen de la fibrilina localizado en el cromosoma 15. Es transmitido en forma autosómica dominante y se diagnostica por el compromiso ocular, esquelético y cardiovascular. El principal riesgo materno en el síndrome de marfan es la disección tipo A, con una mortalidad materna del $22 \%$ durante el reparo. Aún sin disección aórtica el riesgo de disección en mujeres embarazadas con síndrome de Marfan es 1\% (46). La disección aórtica puede ocurrir en cualquier trayecto de la aorta, pero es más frecuente en la porción ascendente. La dilatación de la raíz aórtica mayor a $4,0 \mathrm{~cm}$ identifica a un grupo de riesgo alto con riesgo de disección del $10 \%$, aunque una dimensión normal no descarta la posiblidad de complicaciones. La dilatación de la raíz aórtica puede estar o no acompañada de insuficiencia valvular. Frecuentemente se asocia a prolapso valvular mitral con insuficiencia (17).

Las mujeres con síndrome de Marfan deben evitar quedar embarazadas debido al riesgo de complicaciones (disección y ruptura aórtica). Todas las pacientes con Síndrome de Marfan requieren una ecocardiografía transtorácica con evaluación de las dimensiones de la raíz aórtica. La dilatación mayor de 4,5 cm es indicación de reparo electivo con injerto de tubo con válvula y reimplante de las coronarias antes de la gestación (17) y se recomienda el uso de betabloqueadores durante la gestación. La disección y ruptura son más frecuentes en el tercer trimestre de gestación o cerca al momento del parto. Las pacientes ameritan adecuada analgesia para evitar los incrementos de presión durante el trabajo de parto y el parto y el acortamiento de la segunda fase del trabajo de parto es apropiado. La anestesia general y 
la cesárea pueden permitir un control hemodinámico óptimo.

La corrección quirúrgica exitosa no confiere protección en futuros embarazos, porque la paciente continúa en riesgo de disección aórtica (17).

\section{Paciente con prótesis valvulares}

Las prótesis biológicas pueden presentar degeneración acelerada como consecuencia de la gestación. Sin embargo, la velocidad en el desarrollo de la degeneración es inversamente proporcional a la edad; en algunas ocasiones las mujeres tienen las prótesis valvulares antes del embarazo, y su degeneración durante la gestación es la esperada para este tipo de válvulas. El manejo de pacientes con prótesis biológicas es similar al de mujeres con enfermedad valvular nativa. El embarazo es un estado trombogénico y las pacientes embarazadas con prótesis valvulares mecánicas están en mayor riesgo. El objetivo es continuar con anticoagulación efectiva y segura para la madre y el feto, pero desafortunadamente los medicamentos disponibles no lo hacen $(3,4,47-49)$.

La warfarina (antagonista de la vitamina K) es teratogénica especialmente cuando se usa en el primer trimestre, cruza la barrera placentaria y ha sido asociada con el aumento de la incidencia de abortos espontáneos, partos prematuros y mortinatos. La embriopatía por warfarina tiene una frecuencia entre el $4 \%$ y $10 \%$ y se caracteriza por hipoplasia nasal, alteración de las epífisis, alteraciones oculares y del sistema nervioso central. El riesgo depende de la dosis y es mayor si la exposición ocurre durante las semanas seis y doce de gestación. Durante el embarazo puede llevar a sangrado fetal intracraneal. Las complicaciones fetales son mayores en pacientes tratadas con dosis de warfarina mayores de $5 \mathrm{mg} /$ día. El riesgo de embriopatía puede ser eliminado con la sustitución de warfarina por heparina desde o antes de la semana sexta hasta la semana doce de gestación. La warfarina es relativamente segura durante el segundo y tercer trimestre del embarazo y debe suspenderse y continuar con heparina antes del parto y puede ser utilizada durante la lactancia (50-53).

La heparina no fraccionada (HNF) no cruza la barrera placentaria y es considerada más segura para el feto, ya que no produce sangrado fetal o teratogenicidad. Puede suministrarse en infusión continua o subcutánea, pero es menos segura para la madre debido a la dificultad en lograr adecuada anticoagulación. Además puede ocasionar sangrado en la unión uteroplacentaria y numerosos registros han demostrado una incidencia inaceptable de complicaciones tromboembólicas (frecuencia entre el 12-24\%) que incluyen trombosis valvular fatal en pacientes embarazadas en tratamiento con heparina subcutánea. Cuando la heparina es utilizada durante el primer trimestre, el riesgo de tromboembolismo materno y muerte materna es alto. La eficacia de dosis subcutáneas ajustadas de heparina no ha sido establecida definitivamente. Durante el embarazo, la respuesta de la actividad del tiempo de tromboplastina parcial activado (TTPa) a la heparina está atenuado debido al incremento de los niveles de Factor VIII y fibrinógeno. Dosis ajustadas de HNF subcutáneas pueden causar anticoagulación persistente al momento del parto, lo cual puede complicar su uso antes del parto (54-56).

Las heparinas de bajo peso molecular (HBPM), tienen ventajas sobre la HNF durante el embarazo como: causar menos trombocitopenia, tener una vida media mayor, respuesta más predecible, menor riesgo de osteopenia, fácil administración y al parecer menor riesgo de sangrado. No cruzan la placenta y son seguras para el feto. Al progresar la gestación (y las mujeres aumentar de peso) el volumen de distribución de las HBPM cambia, por lo tanto es necesario medir en plasma los niveles de anti-Xa cuatro a seis horas luego de la dosis de la mañana y ajustar la dosis de HBPM para alcanzar un nivel anti-Xa de aproximadamente 0,71,2 unidades por mililitro. Aunque existe experiencia del uso de HBPM en el tratamiento de la trombosis venosa profunda durante el embarazo, a la fecha no hay estudios adecuados que hayan demostrado su beneficio en pacientes embarazadas con prótesis valvulares cardíacas; incluso existen reportes de trombosis de prótesis valvulares $(14,57,58)$.

Las recomendaciones de la Sociedad Americana del Corazón para anticoagulación en pacientes embarazadas con prótesis mecánicas son (14):

\section{Clase I}

1. Todas las pacientes con prótesis mecánicas deben recibir anticoagulación terapéutica continua con monitoreos frecuentes. (Nivel de evidencia B).

2. Mujeres que requieren anticoagulación a largo plazo con warfarina y están intentando quedar embarazadas, deben realizarse pruebas de emba- 
razo periódicas y definir el tipo de anticoagulación a seguir durante la gestación. La anticoagulación debe continuarse hasta que la paciente esté embarazada. (Nivel de evidencia C).

3. Las pacientes embarazadas con prótesis mecánicas que decidan suspender la warfarina entre la semana seis y doce de la gestación, deben recibir HNF intravenosa continua, HNF con dosis ajustadas o HBPM subcutánea con dosis ajustadas. (Nivel de evidencia C).

4. En pacientes embarazadas con prótesis mecánicas debe discutirse hasta la semana 36 ampliamente las opciones de HNF intravenosa continua ó subcutánea, dosis ajustadas de HBPM ó warfarina. Si se usa HNF intravenosa continua, el riesgo fetal es bajo, pero el riesgo de trombosis de la prótesis valvular, embolización sistémica, infección, osteoporosis y osteopenia inducida por heparina es alto. (Nivel de evidencia C).

5. En pacientes embarazadas con prótesis mecánicas que reciben dosis ajustadas de HBPM, debe ser administrada dos veces al día para mantener un nivel de anti-Xa entre 0,7-1,2 u por ml cuatro horas luego de la administración. (Nivel de evidencia C).

6. En pacientes embarazadas con prótesis mecánicas que reciben dosis ajustadas de HNF, el TTPa debe ser al menos dos veces del control. (Nivel de evidencia C).

7. En pacientes embarazadas con prótesis mecánicas que reciben warfarina; el INR ideal es de 3,0. (Nivel de evidencia $\mathrm{C}$ ).

8. En pacientes embarazadas con prótesis valvulares, la warfarina debe suspenderse y continuar con HNF intravenosa dos ó tres semanas antes del parto. (Nivel de evidencia C).

\section{Clase IIa}

1. En pacientes con prótesis mecánicas, es razonable evitar la warfarina entre la semana 6-12 de gestación para evitar el alto riesgo de malformaciones. (Nivel de evidencia C).

2. En pacientes con prótesis mecánicas, es razonable reiniciar HNF 4-6 horas luego del parto e iniciar warfarina oral en ausencia de sangrado significativo. (Nivel de evidencia C).

3. En pacientes con prótesis mecánicas que reciben anticoagulación con warfarina o heparina, es razonable administrar dosis bajas de aspirina (75100 mg día) en el segundo y tercer trimestre de la gestación. (Nivel de evidencia C).

\section{Conclusiones}

En resumen, las recomendaciones para el manejo de la mujer embarazada con enfermedad valvular, con su respectivo nivel de evidencia según las Guías Europeas de Enfermedad Valvular (59) son:

1. Pacientes con enfermedad valvular tipo estenosis severa deben ser tratadas antes del embarazo, si es posible con tratamiento percutáneo en estenosis mitral. Nivel de evidencia I C.

2. Estudio ecocardiográfico debería realizarse en las pacientes con soplo cardíaco patológico o disnea inexplicada. Nivel de evidencia IC.

3. Pacientes con síndrome de Marfan y diámetro aórtico > $40 \mathrm{~mm}$ debe ser tratadas antes de la gestación. Nivel de evidencia IC.

4. La cirugía con circulación extracorpórea debe ser realizada durante la gestación solo en situaciones donde la vida de la madre corra peligro y no es posible realizar procedimiento percutáneo. Nivel de evidencia IC.

5. El parto vaginal puede realizarse en forma segura en pacientes con enfermedad valvular que se encuentran estables hemodinámicamente. Nivel de evidencia IC.

6. Las pacientes con indicación de anticoagulación puede realizarse con warfarina en el segundo y tercer trimestre hasta la semana 36 de gestación Nivel de evidencia IC.

7. Se requiere estrecho monitoreo de la anticoagulación cuando se utilizan heparinas. Nivel de evidencia IC.

8. La valvuloplastia mitral percutánea debe ser considerada en mujeres embarazadas que tienen síntomas severos o presión sistólica pulmonar mayor de $50 \mathrm{~mm} \mathrm{Hg}$ a pesar de la terapia médica. Nivel de evidencia IlaC.

El manejo de la paciente embarazada con enfermedad valvular debe ser realizado por un grupo multidisciplinario que involucre diferentes áreas como Cardiología, Cardiología Intervencionista, Gineco-Obstetricia, Anestesia cardiovascular y Obstétrica, Cirugía Cardiovascular y Neonatología entre otros

\section{Referencias}

1. Siu S C, Sermer M, Colman J M, Alvarez N et al. Prospective Multicenter Study of Pregnancy Outcomes in Women with Heart Disease. Circulation. 2001;104:515-21. 
2. Siu S C, Colman J M, Sorensen S et al. Adverse Neonatal And Cardiac Outcomes Are More Common In Pregnant Women With Cardiac Disease. Circulation. 2002;105:2179-84.

3. Stout K, Otto C. Pregnancy In Women With Valvular Heart Disease. Heart. 2007;93:552-558.

4. Scirica B, O'Gara P. Valvular Heart Disease In Pregnancy. Current Cardiology Reports. 2006;8:83-89.

5. Gei A, Hankins G. Medical Complications of Pregnancy. Cardiac Disease And Pregnancy. Obstetrics ang Gynecology Clinics of North America. 2001;28(3):465-512.

6. Van Oppen A C, Stigter R H, Bruinse H W. Cardiac Output In Normal Pregnancy: a Critical Review. Obstetrics and Gynaecology. 1996;87:310-18.

7. Easterling T R, Carlson K L, Schmucker B C et al. Measurement Of Cardiac Output In Pregnancy By Doppler Technique. American Journal of Perinatology. 1990;7:220-2.

8. Robson S C, Hunter S, Moore Met al. Haemodynamic Changes During The Puerperium: a Doppler And M-mode Echocardiographic Study. British Journal of Obstetrics and Gynaecology. 1987;94:1028-39.

9. Easterling T R, Benedetti T J, Schmucker B C et al. Maternal Hemodynamics In Normal And Preeclamptic Pregnancies: A Longitudinal Study. Obstetrics and Gynecology. 1990;76:1061-9.

10. De Boer K, Cate J W, Sturk A, Borm J J, Treffers P E. Enhanced Thrombin Generation In Normal And Hypertensive Pregnancy. American Journal of Obstetrics and Gynecology. 1989;160:95-00.

11. Immer F F, Bansi A G, Immer-Bansi A S et al. Aortic Dissection In Pregnancy: Analysis Of Risk Factors And Outcome. The Annals of Thoracic Surgery. 2003;76:309-14.

12. Bryant-Greenwood G D, Schwabe C. Human Relaxins: Chemistry And Biology. Endocrine Reviews. 1994;15:5-26.

13. Warnes C. Pregnancy And Heart Disease. In Braunwald E. Heart disease. A textbook of cardiovascular medicine. 8th ed. Philadelphia. Saunders W B. 2007:p1967

14. Bonow R, Carabello B, Chatterjee Ket al. 2008 Focused Update Incorporated IIn To The ACC/AHA 2006. Guidelines For The Management Of Patients With Valvular Heart Disease. Circulation. 2008;118:523-661.

15. Silversides C, Colman J. Physiological Changes In Pregnancy. In Oakley C, Warnes C. Heart Diseases In Pregnancy. 2nd ed. Blackwell Publishing. 2007.p6.

16. Nihoyannopoulos P. Cardiovascular Examination in Pregnancy and the Approach to Diagnosis of Cardiac Disorder. In Oakley C, Warnes C. Heart Diseases in Pregnancy. 2nd ed. Blackwell Publishing. 2007. p18.

17. Oakley C, Chile A, Jung B et al. Expert Consensus Document of Cardiovascular Diseases During Pregnancy. The Task Force On The Management Of Cardiovascular Diseases During Pregnancy Of The European Society Of Cardiology. European Heart Journal. 2003;24:761-781.

18. Reimold S, Rutherford D. Valvular Heart Disease in Pregnancy. New England Journal Of Medicine. 2003;349:52-9.

19. American Thoracic Society/American College of Chest Physicians. Statement On Cardiopulmonary Exercise Testing. American Journal of Respiratory and Critical Care Medicine. 2003; 167:211-77.

20. Qasqus S A, McPherson C, Frishman W H, Elkayam U. Cardiovascular Pharmacotherapeutic Considerations During Pregnancy and Lactation. Cardiology in Review. 2004;12:201-21.

21. Hameed A, Karaalp IS, Tummala P et al. The Effect of Valvular Heart Disease on Maternal and Fetal Outcome of Pregnancy. Journal of the American College of Cardiology. 2001;37:893-9.

22. Silversides C K, Colman J M, Sermer M, Siu S C. Cardiac Risk in Pregnant Women With Rheumatic Mitral Stenosis. American Journal of Cardiology. 2003;91:1382-5.
23. Zelop C, Heffner L F. The Downside of Cesarean Delivery: Short- and Long-term Complications. Clinical Obstetrics and Gynecology. 2004;47:386 -93.

24. Elkayam U, Gleicher N. Hemodynamics and Cardiac Function During Normal Pregnancy and The Puerperium. In: Elkayam U, Gleicher N. Editors. Cardiac Problems in Pregnancy. New York, NY: Wiley-Liss. 1998;3-22.

25. Wilson W, Taubert KA, Gewitz M, et al. Prevention of infective endocarditis: guidelines from the American Heart Association: a Guideline From The American Heart Association Rheumatic Fever, Endocarditis, and Kawasaki Disease Committee, Council on Cardiovascular Disease in the Young, and the Council on Clinical Cardiology, Council on Cardiovascular Surgery and Anesthesia, and the Quality of Care and Outcomes Research Interdisciplinary Working Group. Circulation2007;116:1736-54.

26. Baddour L M, Bettmann MA, Bolger A F et al. Nonvalvular cardiovascular device-related infections. Circulation 2003;108:2015-31.

27. Warnes C A, Williams R G, Bashore T M et al. ACC/AHA. 2008. Guidelines For The Management of Adults With Congenital Heart Disease: A Report of The American College of Cardiology/American Heart Association Task Force on Practice Guidelines (Writing Committee to Develop Guidelines for the Management of Adults With Congenital Heart Disease). Journal of The American College of Cardiology. 2008.

28. Elkayam U, Bitar F. Valvular Heart Disease and Pregnancy. Part I: Native Valves. Journal of The American College of Cardiology. 2005;46:223-230.

29. Boggess K A, Watts D H, Hillier S L et al. Bacteremia Shortly After Placental Separation During Cesarean Delivery. Obstetrics and Gynaecology. 1996;87:779-784.

30. Campuzano K, Roque H, Bolnick A et al. Bacterial Endocarditis Complicating Pregnancy: Case Report and Systematic Review of the Literature. Archives of Gynecology and Obstetrics. 2003, 268:251-255.

31. Hameed A, Karaalp I S, Tummala P P et al. The Effect of Valvular Heart Disease on Maternal and Fetal Outcome of Pregnancy. Journal of the American College of Cardiology. 2001, 37:893-899.

32. Silversides C K, Colman J M, Sermer M, Siu S C. Cardiac Risk in Pregnant Women With Rheumatic Mitral Stenosis. American Journal of Cardiology. 2003;91:1382-1385.

33. Barbosa PJ, Lopes AA, Feitosa GS et al. Prognostic Factors of Rheumatic Mitral Stenosis During Pregnancy and Puerperium. Arquivos Brasileiros de Cardiologia 2000;75:215-224.

34. Weiss B M, Von Segesser L K, Alon E et al. Outcome of Cardiovascular Surgery and Pregnancy: A Systematic Review of the Period 1984-1996. American Journal of Obstetrics and Gynecology. 1998;179:1643-1653.

35. De Souza J A, Martinez E E Jr, Ambrose J A et al. Percutaneous Balloon Mitral Valvuloplasty in Comparison with Open Mitral Valve Commissurotomy for Mitral Stenosis During Pregnancy. Journal of the American College of Cardiology. 2001;37:900-903.

36. Reimold S C, Rutherford J D. Clinical Practice. Valvular Heart Disease in Pregnancy. New England Journal Of Medicine. 2003, 349:52-59.

37. Bernard L. Mitral Valve Prolapsed. In Oakley C, Warnes C. Heart Diseases in Pregnancy. 2 ed. Blackwell Publishing. 2007. p96.

38. Lao T T, Adelman A G, Sermer M, Colman J M. Balloon Valvuloplasty for Congenital Aortic Stenosis n Pregnancy. British Journal Obstetrics and Gynecology. 1993;100:1141-2.

39. Banning A P, Pearson J F, Hall R J. Role of Balloon Dilatation of the Aortic Valve in Pregnant Patients with Severe Aortic Stenosis. British Heart Journal. 1993;70:544-5. 
40. Otto C M. Timing of Aortic Valve Surgery. Heart. 2000;84:211218.

41. Mohler E R III. Mechanisms of Aortic Valve Calcification. American Journal of Cardiology. 2004;94:1396-1402.

42. Sheikh F, Rangwala S, DeSimone C, Smith HS, O'Leary A M. Management of the Parturient With Severe Aortic Incompetence. Journal of Cardiothoracic and Vascular Anesthesia. 1995;9:575-7.

43. Veldtman G R, Connolly H M, Grogan M et al. Outcomes of Pregnancy in Women with Tetralogy of Fallot. Journal of the American College of Cardiology. 2004;44:174-80.

44. Meijer J M, Pieper P G, Drenthen W et al. Pregnancy, Fertility, and Recurrence Risk in Corrected Tetralogy of Fallot. Heart. 2005;91:801-5.

45. Thorne S A. Congenital Heart Disease, Pregnancy in the Heart Disease. Heart. 2004;90:450-456

46. Sadler L, McCowan L, White $\mathrm{H}$ et al. Pregnancy Outcomes and Cardiac Complications in Women with Mechanical, Bioprosthetic and Homograft Valves. An International Journal of Obstetrics and Gynaecology. 2000;107:245-253.

47. Trimm J, Hung L, Rahimtoola S. Artificial heart valves. En: Oakley C, Warnes C. Heart diseases in pregnancy. 2 ed. Blackwell Publishing. 2007:104.

48. Salazar E, Espinola N, Roman L, Casanova J M. Effect of Pregnancy on the Duration of Bovine Pericardial Bioprostheses. American Heart Journal. 1999;137:714-720.

49. Sbarouni E, Oakley C M. Outcome of Pregnancy in Women with Valve Prostheses. British Heart Journal. 1994;71:196-201.
50. Hung L, Rahimtoola S H. Prosthetic Heart Valves and Pregnancy. Circulation 2003;107:1240-6.

51. Wong V, Cheng C H, Chan K C. Fetal and Neonatal Outcome of Exposure to Anticoagulants during Pregnancy. American Journal of Medical Genetics. 1993;45:17-21.

52. Hirsh J, Fuster V, Ansell J, Halperin J L. American Heart Association/American College of Cardiology Foundation Guide to Warfarin Therapy. Journal of The American College of Cardiology. 2003;41:1633-52.

53. Hirsh J, Fuster V. Guide to Anticoagulant Therapy, Part 2. Oral Anticoagulants. American Heart Association. Circulation. 1994;89:01469-80.

54. Salazar E, Izaguirre R, Verdejo J, Mutchinick O. Failure of Adjusted Doses of Subcutaneous Heparin to Prevent Thromboembolic Phenomena in Pregnant Patients with Mechanical Cardiac Valve Prostheses. Journal of the American College of Cardiology. 1996;27:1698-703.

55. Ginsberg J S, Hirsh J. Use of Antithrombotic Agents during Pregnancy. Chest. 1995;108:305S-11S.

56. Elkayam U R. Anticoagulation in Pregnant Women with Prosthetic Heart Valves: a Double Jeopardy. Journal of the American College of Cardiology. 1996;27:1704-6.

57. Ginsberg J S, Chan W S, Bates S M, Kaatz S. Anticoagulation of Pregnant Women with Mechanical Heart Valves. Archives of Internal Medicine. 2003;163:694-8.

58. Vahanian A, Baumgartner $\mathrm{H}, \mathrm{Bax} \mathrm{J}$ et al. Guidelines on the management of valvular heart disease The Task Force on the Management of Valvular Heart Disease of the European Society of Cardiology European Heart Journal (2007) 28, 230-268. 